\title{
Prevalencia de istmocele en el Hospital de San José de Bogotá, Colombia
}

\author{
Oscar Benedetti F. ${ }^{1}$, Catalina Agudelo R. ${ }^{1}$, Rodrigo Rodríguez R. ${ }^{1}$, Ángel Miranda C. ${ }^{1}$, \\ Henry Rodríguez D. ${ }^{1}$, Carlos Castro C. ${ }^{1}$ \\ ${ }^{1}$ Especialista en Cirugía Endoscópica Ginecológica. Fundación Universitaria de Ciencias de la Salud-Hospital de San \\ José. Bogotá, Colombia.
}

\section{RESUMEN}

Objetivo: Describir la prevalencia de istmocele como hallazgo incidental en pacientes con antecedente de cesárea y síntomas clínicos asociados. Métodos: Estudio descriptivo de corte transversal, mediante muestreo no probabilístico por conveniencia, en pacientes con antecedente de cesárea, programadas para histeroscopia entre noviembre de 2014 y marzo de 2015, en el servicio de cirugía endoscópica ginecológica del Hospital San José de Bogotá, Colombia. Resultados: 42 pacientes fueron elegibles para el estudio por su antecedente de cesárea y todas fueron incluidas. La frecuencia de istmocele fue $83,3 \%$ en la histeroscopia, con similar localización en istmo y cérvix. La principal característica clínica presentada por las pacientes fue hemorragia uterina anormal $(85,7 \%)$, mientras que la menos frecuente fue infertilidad $(7,1 \%)$. En las pacientes con presencia de istmocele se observó una mayor prevalencia de dismenorrea $(65,7 \%$ vs. $42,9 \%)$, antecedente de 2 o más cesáreas $(60 \%$ vs. $42,9 \%)$ y cesárea de urgencia $(54,3 \%$ vs. $28,6 \%$ ) comparadas con el grupo de pacientes sin istmocele, en este último grupo se advirtió que el $100 \%$ de las pacientes no tenían antecedente de trabajo de parto previo. En mujeres con antecedente de cesárea y presencia de síntomas como sangrado uterino anormal, dismenorrea, dolor pélvico, infertilidad y dispareunia, la frecuencia de istmocele diagnosticado por histeroscopia fue mayor del 80\%. Conclusión: El istmocele se debe a la cicatrización anómala uterina posterior a una cesárea, se requieren otros estudios para determinar no solo la prevalencia sino los factores protectores que reduzcan su incidencia para tener un impacto positivo en este tipo de pacientes.

\section{PALABRAS CLAVE: Istmocele, histeroscopia, cesárea, ultrasonografía}

\section{SUMMARY}

Objective: To describe the prevalence of isthmocele as an incidental finding in patients with a history of cesarean section and associated clinical symptoms. Methods: Descriptive cross-sectional study using nonprobabilistic sampling for convenience in patients with a history of cesarean section, scheduled for hysteroscopy between November 2014 and March 2015, in the gynecological endoscopic surgery service of the Hospital San José de Bogotá, Colombia. Results: 42 patients were eligible for the study because of their previous cesarean section and all were included. The frequency of isthmocele was $83.3 \%$ in hysteroscopy, with similar localization in the isthmus and cervix. The main clinical characteristic presented by the patients was abnormal uterine bleeding ( $85.7 \%)$, while the less common was infertility $(7.1 \%)$. A higher prevalence of dysmenorrhea (65.7\% vs. $42.9 \%$ ) was observed in patients with isthmocele, a history of 2 or more cesareans $(60 \%$ vs. $42.9 \%)$ and an emergency cesarean section $(54,3 \%$ vs. $28.6 \%)$ compared to the group of patients without isthmocele, in the latter group it was noted that $100 \%$ of the patients had no previous history 
of labor. In women with a history of cesarean section and presence of symptoms such as abnormal uterine bleeding, dysmenorrhea, pelvic pain, infertility and dyspareunia, the frequency of isthmocele diagnosed by hysteroscopy was greater than $80 \%$. Conclusion: Isthmocele is due to abnormal uterine cicatrization after cesarean section, other studies are required to determine not only the prevalence but also the protective factors that reduce its incidence to have a positive impact on this kind of patients.

\section{KEY WORDS: Isthmocele, hysteroscopy, cesarean section, ultrasonography}

\section{INTRODUCCIÓN}

El istmocele se ha considerado causa de infertilidad y sangrado uterino anormal. Fue descrito en 1995 por Morris, quien estudió las piezas quirúrgicas de 51 histerectomías en mujeres que tuvieron cesárea anterior con el fin de determinar los hallazgos histopatológicos presentes en la cicatriz de la cesárea (1), sin embargo, aún existe controversia en cuanto a su definición, incluso se han adoptado varios términos como nicho, divertículo, bolsa, cicatriz de cesárea deficiente, defecto de cicatriz, a esto se suma la falta de consenso para los criterios diagnósticos y la no disponibilidad de un patrón de oro aceptado por todos los autores para la realización de comparaciones (2-8). Gubbini y cols (9) lo definen como un defecto de la cara anterior del útero, en la cicatriz de una cesárea anterior ubicado a nivel ístmico o cervical dependiendo de si hubo o no trabajo de parto previo a la cesárea.

La prevalencia reportada en pacientes con antecedentes de cesárea oscila entre 56\% a $84 \%$, variando en función del método de detección, los criterios utilizados para la definición de istmocele y la población de estudio (2). Se han descrito diferentes factores de riesgo asociados a la presentación de istmocele principalmente el número de cesáreas previas, localización de la cicatriz, trabajo de parto antes de la cesárea, posición del útero entre otros (2).

No se conocen con claridad cuáles son los mecanismos que producen el defecto de la cicatrización de la cesárea anterior $(8,10,11)$. Se cree que la técnica quirúrgica utilizada para la histerorrafia en la cesárea, sobre todo cuando se utiliza el cierre en un solo plano, puede guardar relación con la etiología $(4,8,11)$. También se encuentra posiblemente implicada el tipo de sutura que se utilice, la inflamación crónica y la particularidad inherente de cada persona en los procesos de cicatrización (8).

El síntoma principal de esta patología es el sangrado uterino anormal o manchado postmenstrual y se encuentra en la mayoría de las pacientes con cesárea previa $(2,4,7,8,12)$. Otros síntomas menos frecuentes son la dismenorrea, dolor pélvico, dispareunia e infertilidad secundaria $(13,14)$. Respecto a este último, se ha demostrado que la pseudocavidad presente en el istmocele, causa un sangrado uterino posmenstrual por acúmulo de sangre y restos endometriales retenidos que van fluyendo lentamente, ocasionando una perturbación en el moco cervical, alterando el transporte y calidad de los espermatozoides e interferencia en la implantación $(11,15)$, aunque también muchas mujeres con istmocele pueden permanecer asintomáticas (16).

Gran parte de los estudios definen el istmocele como un defecto triangular o adelgazamiento del miometrio, identificado con ecografía transvaginal o histerosonografía $(2,4,9,16,17)$. Actualmente, no existe un consenso ni una prueba de oro para diagnosticar el istmocele (2), sin embargo, la ecografía transvaginal es el método más comúnmente utilizado para el diagnóstico desde $1990(4,9,11)$, seguida de la histerosonografía, que detecta el defecto en todas las pacientes con antecedente de cesárea según el estudio de Monteagudo y cols (17). Otros métodos utilizados en menor proporción son la histerosalpingografía y la histeroscopia, esta última cada día adquiere mayor auge ya que ha reportado una detección del $100 \%$ de pacientes con istmocele identificado previamente por ecografía, adicionalmente ayuda a descartar otras causas de imagen anecoica en la cicatriz, y permite la visualización directa del defecto y su corrección $(2,4,18-21)$

El tratamiento del istmocele mediante la resección histeroscópica del anillo fibroso o istmoplastia con o sin ablación del tejido endometrial ectópico de la fosa del istmo fue descrito por primera vez por Fabres y cols (22). De ahí en adelante se han propuesto varias técnicas entre las que están el manejo laparoscópico, vaginal y combinado (laparoscópico y vaginal) $(18,23,24,25)$. También han surgido la resección laparoscópica asistida por robot y la terapia hormonal para control del dolor pélvico y sangrado uterino anormal, sin embargo, los resultados de los estudios relacionados con estos tipos de manejo son contradictorios $(11,18,23,26)$.

Con el aumento de la incidencia de cesáreas en nuestro medio (27) el objetivo de esta comunicación es conocer la prevalencia de istmocele como hallazgo incidental en pacientes con antecedente de cesárea y describir sus características clínicas. 


\section{PACIENTES Y MÉTODOS}

Se llevó a cabo un estudio descriptivo de corte transversal. Las participantes fueron seleccionadas mediante muestreo no probabilístico por conveniencia. En el periodo comprendido entre noviembre de 2014 y marzo de 2015, se incluyeron todas las pacientes a quienes se les realizó histeroscopia diagnóstica o terapéutica por cualquier indicación, con antecedente de una o más cesáreas atendidas en el servicio de cirugía endoscópica ginecológica del Hospital de San José (HSJ), institución de tercer nivel y centro de referencia para pacientes con patologías ginecológicas que requieren cirugía endoscópica avanzada.

Los datos fueron obtenidos durante la consulta de programación de la cirugía mediante un formato de recolección previamente diseñado. Se registraron datos relacionados con edad, paridad, número de cesáreas, posición del útero, antecedentes de trabajo de parto previo a la cesárea, ruptura prematura de membranas, cesárea de urgencia, infertilidad, dolor pélvico crónico, hemorragia uterina anormal, dismenorrea, dispareunia e istmocele reportado en ecografía previa, tomado como un defecto en la pared anterior del útero en el sitio de la cicatriz de una cesárea previa, de forma triangular y con base hacia cavidad uterina (11).

Las pacientes fueron informadas sobre el proyecto de investigación y se solicitó su consentimiento informado escrito para participar en el estudio. Este estudio fue aprobado por el Comité de Ética e Investigación de la Fundación Universitaria de Ciencias de la Salud (FUCS).

Procedimiento: La histeroscopia se realizó siguiendo el protocolo institucional, bajo anestesia general y utilizando solución salina al 0,9\% como medio de distensión (Figura 1), durante su realización se evaluó la presencia o no de istmocele tomando como criterio diagnóstico de éste cualquier defecto de la cara anterior del útero, en la cicatriz de una cesárea anterior ubicado a nivel ístmico o cervical dependiendo de si hubo o no trabajo de parto previo (11). En aquellas pacientes en las que se descartó la causa principal que indicó la realización de la histeroscopia y que presentaban istmocele asociado a sangrado uterino anormal, dolor pélvico o infertilidad, se procedió a la corrección quirúrgica del mismo utilizando asa de resectoscopio con energía bipolar (Figura 2). Todas las pacientes fueron citadas para la valoración posoperatoria y revisión del resultado de patología 15 días después del procedimiento.

Análisis estadístico: El análisis descriptivo se realizó en términos de frecuencias absolutas y relativas para las variables categóricas, y por medio de medianas y rangos intercuartílicos para las variables cuantitativas. Los datos se analizaron utilizando el programa Stata $13 \AA$.

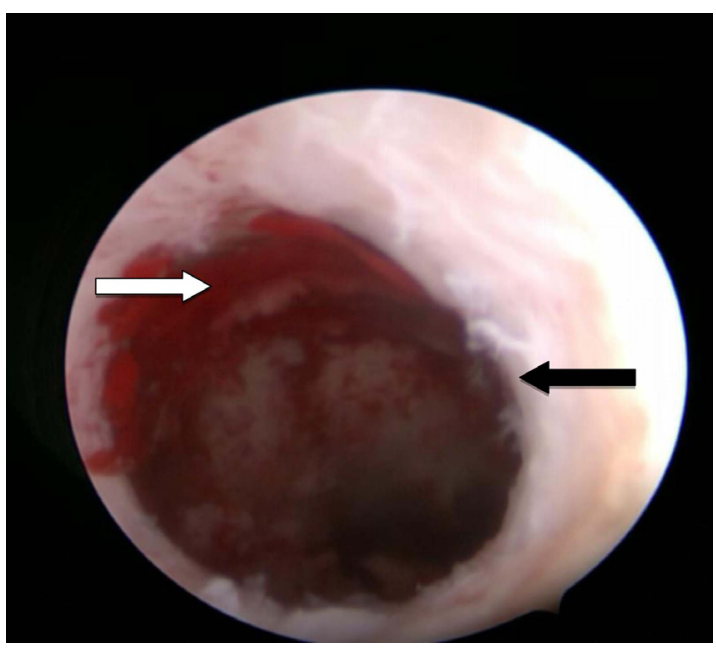

Figura 1. Histeroscopia. Utilizando solución salina al 0,9\% como medio de distensión, se observa orificio cervical interno (flecha negra) e istmocele (flecha blanca).

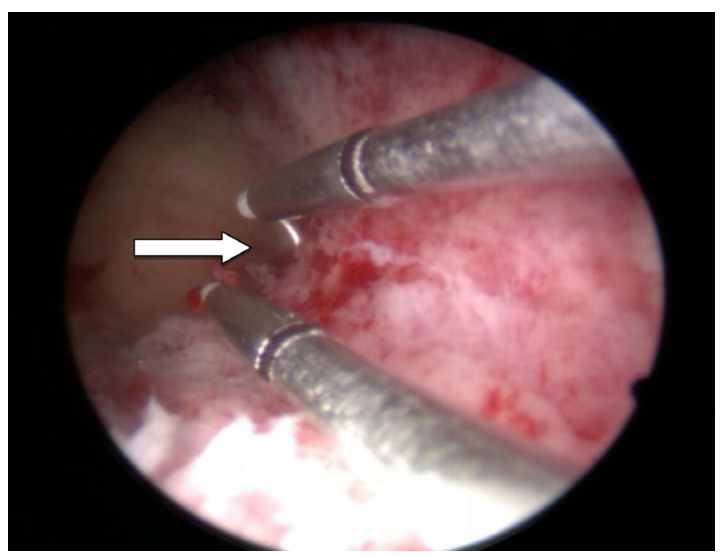

Figura 2. Histeroscopia. Utilizando solución salina al 0,9\% como medio de distensión, se observa remodelación de istmocele con asa de resectoscopio bipolar (flecha blanca).

\section{RESULTADOS}

Durante noviembre de 2014 y marzo de 2015 fueron llevadas a histeroscopia un total de 195 pacientes en el servicio de Cirugía Endoscópica Ginecológica del HSJ, de éstas 42 pacientes eran elegibles para el estudio por su antecedente de cesárea y fueron todas incluidas, las variables a describir 
hacen parte de campos obligatorios en el diligenciamiento de la historia clínica de la institución por lo cual se tuvo disponibilidad de la totalidad de estos datos para cada paciente. Todas las pacientes contaban con reporte ecográfico previo a la programación de la cirugía. La mediana de edad fue 41,5 años (RIQ: 35 a 47), el 50\% de las mujeres tenía antecedente de 2 partos previos. Se categorizó la variable número de cesáreas previas en 2 grupos: de 1 a 2 y de 3 a 4 cesáreas, ya que la literatura ha reportado mayor riesgo de istmocele a mayor número de cesáreas previas (12), 36 (85,7\%) participantes se encontraron en el primero grupo y 6 $(14,3 \%)$ en el segundo.

El principal síntoma presentado por las pacientes fue la hemorragia uterina anormal $(85,7 \%)$ mientras que el menos frecuente fue la infertilidad $(7,1 \%)$. Del total de 42 pacientes, 21 (50\%) habían sido llevadas a cesárea de urgencia y $17(40,5 \%)$ presentaron trabajo de parto previo a la cesárea. En cuanto a la posición del útero, en 31 (73,8\%) se encontraba en anteversoflexón mientras que en las $11(26,2 \%)$ pacientes restantes se encontraba en retroversoflexión. La Tabla I presenta una descripción detallada de estas características.

En 35 (83,3\%) de las 42 pacientes se evidenció la presencia de istmocele en la histeroscopia, de éstas, 2 (5,7\%) pacientes tenían reporte de istmocele en la ecografía. La frecuencia de localización del istmocele fue similar tanto en el istmo como en el cérvix $(48,6 \%$ y $51,4 \%$ respectivamente).

Al analizar la frecuencia de las características clínicas según la presencia o no de istmocele (Tabla II), se encontró que la frecuencia de éstas fue similar en ambos grupos. Sin embargo, en las pacientes con presencia de istmocele se observó una mayor prevalencia de dismenorrea $(65,7 \%$ vs. $42,9 \%$ ), antecedente de 2 o más cesáreas (60\% vs. $42,9 \%)$ y cesárea de urgencia $(54,3 \%$ vs. $28,6 \%)$ comparadas con el grupo de pacientes sin istmocele. Las 7 pacientes que no presentaron istmocele tuvieron en común que ninguna de ellas había presentado trabajo de parto previo a cesárea, mientras que en el grupo con istmocele, ubicado ya fuera en el istmo o en el cérvix, el porcentaje de trabajo de parto previo fue $48,6 \%$. De las 11 pacientes con úteros en retroversoflexion, 10 presentaron istmocele.

\section{DISCUSIÓN}

Teniendo en cuenta que en Colombia aún no hay estudios sobre istmocele, este estudio se planteó para revisar su frecuencia en mujeres llevadas a histeroscopia con antecedente de cesárea en nuestro medio y describir las características clínicas de estas pacientes.

\section{Tabla I \\ CARACTERÍSTICAS CLÍNICAS DE LA MUESTRA DE PARTICIPANTES}

\begin{tabular}{lr}
\hline Variable & $\mathrm{n}(\%)$ \\
\hline Paridad: & \\
1 & $7(16,7)$ \\
2 & $21(50,0)$ \\
3 & $9(21,4)$ \\
4 & $4(9,5)$ \\
7 & $1(2,4)$
\end{tabular}

Istmocele:

Presencia en histerosocopia $35(83,3)$

Presencia en ecografía $2(4,8)$

Localización:

Istmo uterino

$17(48,6)$

Cérvix

$18(51,4)$

Síntomas:

Hemorragia uterina anormal $\quad 36(85,7)$

Dismenorrea $26(61,9)$

Dispareunia $17(40,5)$

Dolor pélvico crónico $14(33,3)$

Infertilidad $\quad 3(7,1)$

Factores de riesgo:

Número de cesáreas

2

3

4

Trabajo de parto previo

Ruptura prematura de membranas $\quad 8(19,0)$

Cesárea de urgencia $21(50,0)$

Posición del útero:

Anteversoflexion $\quad 31(73,8)$

Retroversoflexion $11(26,2)$

En nuestro estudio, la frecuencia de istmocele por histeroscopia fue del $83,3 \%$. Se consideró istmocele, según lo publicado por Gubbini y cols (9), como un defecto de la cara anterior del útero, en la cicatriz de una cesárea anterior ubicado a nivel ístmico o cervical dependiendo de si hubo o no trabajo de parto previo a la cesárea. Los síntomas que predominaron en orden de frecuencia fueron hemorragia uterina anormal y dismenorrea. Adicionalmente, se advirtió que el $100 \%$ de las pacientes sin istmocele no reportaron antecedente de trabajo de parto previo. Estos datos se asemejan a los publicados por otros autores donde también mencionan estas características como factores de riesgo para la presentación de istmocele $(1,2,4,7,8,16,28)$. 


\section{Tabla II}

FRECUENCIA DE LAS CARACTERÍSTICAS CLÍNICAS SEGÚN LA PRESENCIA DE ISTMOCELE

\begin{tabular}{|c|c|c|}
\hline \multirow[b]{2}{*}{ Variable } & \multicolumn{2}{|c|}{ Istmocele } \\
\hline & $\begin{array}{c}\mathrm{Si} \\
\mathrm{n}=35(83,3 \%)\end{array}$ & $\begin{array}{c}\text { No } \\
n=7(16,7 \%)\end{array}$ \\
\hline \multicolumn{3}{|c|}{ Hemorragia uterina anormal } \\
\hline $\mathrm{Si}$ & $30(85,7)$ & $6(85,7)$ \\
\hline No & $5(14,3)$ & $1(14,3)$ \\
\hline \multicolumn{3}{|l|}{ Dismenorrea } \\
\hline $\mathrm{Si}$ & $23(65,7)$ & $3(42,9)$ \\
\hline No & $12(34,3)$ & $4(57,1)$ \\
\hline \multicolumn{3}{|l|}{ Dispareunia } \\
\hline $\mathrm{Si}$ & $14(40,0)$ & $3(42,9)$ \\
\hline No & $21(60,0)$ & $4(57,1)$ \\
\hline \multicolumn{3}{|c|}{ Dolor pélvico crónico } \\
\hline $\mathrm{Si}$ & $11(31,4)$ & $3(42,9)$ \\
\hline No & $24(68,6)$ & $4(57,1)$ \\
\hline \multicolumn{3}{|l|}{ Infertilidad } \\
\hline $\mathrm{Si}$ & $2(5,7)$ & $1(14,3)$ \\
\hline No & $33(94,3)$ & $6(85,7)$ \\
\hline \multicolumn{3}{|c|}{ Número de cesáreas } \\
\hline 1 & $14(40,0)$ & $4(57,1)$ \\
\hline 2 & $15(42,9)$ & $3(42,9)$ \\
\hline 3 & $5(14,3)$ & $0(0,00)$ \\
\hline 4 & $1(2,9)$ & $0(0,00)$ \\
\hline \multicolumn{3}{|c|}{ Trabajo de parto previo } \\
\hline $\mathrm{Si}$ & $17(48,6)$ & $0(0,0)$ \\
\hline No & $18(51,4)$ & $7(100,0)$ \\
\hline \multicolumn{3}{|c|}{ Ruptura prematura de membranas } \\
\hline $\mathrm{Si}$ & $7(20,0)$ & $1(14,3)$ \\
\hline No & $28(80,0)$ & $6(85,7)$ \\
\hline \multicolumn{3}{|c|}{ Cesárea de urgencia } \\
\hline $\mathrm{Si}$ & $19(54,3)$ & $2(28,6)$ \\
\hline No & $16(45,7)$ & $5(71,4)$ \\
\hline \multicolumn{3}{|l|}{ Posición del útero } \\
\hline Anteversoflexion & $25(71,4)$ & $6(85,7)$ \\
\hline Retroversoflexion & $10(28,6)$ & $1(14,3)$ \\
\hline
\end{tabular}

En 2014, Bij de Vaate y cols (2), publican la revisión más grande sobre istmocele encontrada en la literatura, en este artículo reportan una prevalencia que va del $19,4 \%$ al $88 \%$, datos que concuerdan con lo registrado por nosotros. Entre los estudios incluidos en la revisión, los métodos de detección reportados fueron ecografía transvaginal, histerosonografías o histeroscopia, siendo éste último el método diagnóstico utilizado en el presente estudio.
La prevalencia tan baja por ecografía $(4,8 \%)$ en nuestra serie difiere mucho de la encontrada en otros estudios como el de Armstrong y cols (3) en 2003, quienes evaluaron la capacidad de la ecografía transvaginal para detectar cicatrices de cesárea y sus defectos en mujeres no embarazadas, encontrando una identificación de cicatrices de cesárea en tiempo real con una sensibilidad del $100 \%$ (IC $95 \% 88,8-100$ ) y una especificidad del $100 \%$ (IC $95 \% 90,7-100)$, pero advierten que al revisar las 
imágenes almacenadas, la cicatriz no se identificó en 4 casos (12,9\%), dando una sensibilidad del $87,1 \%$ y una especificidad del $100 \%$ (kappa $=0,88$ IC 95\% 0,77-0,99), acuerdo sustancial entre imágenes en tiempo real y la revisión de la imagen almacenada (3). En ese mismo estudio, encontraron el defecto de la cicatriz de cesárea en 13 pacientes $(42 \%)$ con la ecografía transvaginal, todas las pacientes habían tenido trabajo de parto previo antes de la cesárea $(p=0,01)$ y un mayor número de cesáreas que las que no tenían istmocele por ecografía $(p<0,04)(3)$.

Otro estudio del que difieren nuestros hallazgos en cuanto a la prevalencia de istmocele por ecografía es el de Bij de Vaate y cols (28), quienes realizaron un trabajo observacional descriptivo con seguimiento de 6 a 12 meses mediante ecografía transvaginal a 225 mujeres que habían tenido parto por cesárea, en busca de istmocele, observándose el defecto en 54 pacientes (24\%). También podemos referenciar a Osser y cols (6), quienes estimaron la prevalencia de los defectos de la cicatriz de cesárea mediante ecografía transvaginal, en un seguimiento con este método de 6 a 9 meses posterior al parto en 108 mujeres, que se habían sometido a una cesárea y se visualizó la cicatriz en el $100 \%$ y defecto de la misma en 66 pacientes (61\%).

Podríamos decir que la frecuencia de istmocele evidenciada por histeroscopia en nuestro estudio se debió correlacionar con una frecuencia similar en la ecografía transvaginal, ya que varias series afirman que la ecografía transvaginal puede detectar con precisión las cicatrices de cesárea y por ende sus defectos (29). Además, según Fabres y cols (4), la correlación entre ambas técnicas en el diagnóstico de istmocele es del $100 \%$, dato que no se vió reflejado en nuestros resultados por falta de reporte ecográfico de este hallazgo, sin embargo, aún no es claro si este subregistro se deba a la falta de visualización del defecto por parte de los ecografistas o si se ha visualizado como un hallazgo normal y por esto no se reporta.

Sobre los antecedentes de las pacientes con istmocele, Osser y cols (6), Ofili-Yebovi y cols (30), Wang y cols (7) y Zimmer y cols (29), registran como factores de riesgo más prevalentes el útero en retroversoflexión, la cesárea de urgencia y la localización de la cicatriz. En nuestro estudio, 19 de las 21 pacientes con cesárea de urgencia tenían istmocele y 10 de las 11 pacientes con útero en retroversoflexión también presentaban este defecto en la cicatriz. Esto no es sorprendente y puede ser razonable o explicado por la alteración en el proceso de curación de la histerotomía, secundario a factores como la tensión mecánica del segmento uterino inferior cuando este se encuentra en retroflexión, causando un déficit en la perfusión sanguínea y la oxigenación, afectando la cicatrización de las heridas (28). De igual forma sucede cuando la localización de la histerotomía se encuentra muy baja, donde las propiedades curativas del cuello uterino, que pudiera estar incluido en la sutura de cierre, podrían ser menos favorables que las del miometrio en el istmo o cuerpo del útero (28). Esto es observado con más frecuencia cuando existe trabajo de parto antes de la cesárea (28). Por la tendencia observada en nuestros hallazgos, es posible que las diferencias hubiesen sido más evidentes con un número mayor de pacientes.

En nuestro estudio, Ilama la atención que el antecedente de trabajo de parto previo a la cesárea solo se encontró presente en mujeres en quienes se confirmó la presencia de istmocele. Armnstrong y cols (3), hallaron en su estudio que el $100 \%$ de las pacientes que tenían istmocele tenían antecedente de trabajo de parto antes de la cesárea. Si bien es cierto que en el grupo que se evidenció istmocele en nuestro trabajo el $48 \%$ tenían antecedente de trabajo de parto antes de la cesárea y el $51 \%$ no, el $100 \%$ del grupo en el que no se evidenció istmocele carecían de tal antecedente. Siendo este antecedente importante como posible factor que favorecería la presentación de la patología estudiada.

Dentro de nuestro hallazgos se registró que el síntoma predominante fue la hemorragia uterina anormal (85,7\%) seguido de dismenorrea $(61,9 \%)$, estos resultados son similares a lo reportado por Bij de Vaate y cols y otros autores como Fabres y cols, quienes concluyen que los trastornos del sangrado menstrual pueden estar relacionados con la presencia de un istmocele en ausencia de otras entidades patológicas, sugiriendo este defecto anatómico como una posible causa $(2,4)$. Este dato está en estrecha relación con lo reportando también por Wang y cols (7), quienes demostraron que el sangrado uterino anormal manifestado por pacientes como manchado postmenstrual prolongado fue el síntoma más común $(63,8 \%, 131 / 207)$, seguido por dismenorrea $(53,1 \%, 108 / 207)$, dolor pélvico crónico $(39,6 \%, 82 / 207)$ y dispareunia $(18,3 \%, 38 / 207)$.

Los resultados del presente estudio aportan información relevante en un área que no está bien dilucidada en la literatura, con escasas publicaciones que tratan de determinar los factores de riesgo que pueden estar asociados a la patología y a su detección por ecografía. Una de las fortalezas fue la recolección prospectiva de la muestra, lo cual evita la subestimación de la frecuencia de istmocele en historias clínicas anteriores a la realización de este trabajo.

La principal debilidad del estudio es el tamaño pequeño de la muestra, además, la frecuencia de síntomas como hemorragia uterina anormal y dismenorrea pudieron estar sesgadas ya que algunas pacientes tenían otras patologías como pólipos, miomas, hiperplasia endometrial, DIU, etc. que 
pueden comportarse como un factor de confusión en las pacientes que no presentaban istmocele y manifestaron los síntomas, así mismo no se puede atribuir de forma confiable al istmocele ser el causante de los síntomas si la paciente tenía otras condiciones patológicas.

Nuestros resultados proporcionan la base para nuevos estudios acerca de la importancia clínica de los defectos de la cicatriz de cesárea incluyendo la posible asociación con factores de riesgo y mecanismos descritos en la literatura, pero que no se ha comprobado su causalidad, dentro de los cuales se encuentra la técnica de cierre de la histerorrafia y que no incluimos en este trabajo por la imposibilidad de confirmación de esta variable.

\section{CONCLUSIONES}

De las 42 pacientes estudiadas, 83,3\% presentaron istmocele diagnosticado por histeroscopia. Es importante que ante síntomas como el sangrado uterino anormal, dismenorrea, dolor pélvico, infertilidad y dispareunia, tanto ginecólogos como radiólogos, consideren esta entidad, como posible causa y diagnóstico diferencial, teniendo en cuenta que es un defecto fácil de diagnosticar y corregir. Se necesitan estudios con diseños analíticos y tamaños de muestra adecuados para establecer la asociación entre estos factores con los defectos de la cicatriz de cesárea.

\section{REFERENCIAS}

1. Morris H. Surgical pathology of the lower uterine segment caesarean section scar: is the scar a source of clinical symptoms? Int J Gynecol Pathol. 1995;14(1):16-20.

2. Bij de Vaate AJ, van der Voet LF, Naji O, Witmer M, Veersema S, Brolmann HA, et al. Prevalence, potential risk factors for development and symptoms related to the presence of uterine niches following cesarean section: systematic review. Ultrasound Obstet Gynecol. 2014;43(4):372-82.

3. Armstrong V, Hansen WF, Van Voorhis BJ, Syrop $\mathrm{CH}$. Detection of cesarean scars by transvaginal ultrasound. Obstet Gynecol. 2003;101(1):61-5.

4. Fabres C, Aviles G, De La Jara C, Escalona J, Munoz JF, Mackenna A, et al. The cesarean delivery scar pouch: clinical implications and diagnostic correlation between transvaginal sonography and hysteroscopy. J Ultrasound Med. 2003;22(7):695-700.

5. Surapaneni K, Silberzweig JE. Cesarean section scar diverticulum: appearance on hysterosalpingography. AJR Am J Roentgenology. 2008;190(4):870-4.

6. Osser OV, Jokubkiene L, Valentin L. High prevalence of defects in Cesarean section scars at transvaginal ultrasound examination. Ultrasound Obstet Gynecol. 2009;34(1):90-7.

7. Wang CB, Chiu WW, Lee CY, Sun YL, Lin YH, Tseng CJ. Cesarean scar defect: correlation between cesarean section number, defect size, clinical symptoms and uterine position. Ultrasound Obstet Gynecol. 2009;34(1):85-9.

8. Borges LM, Scapinelli A, de Baptista Depes D, Lippi UG, Coelho Lopes RG. Findings in patients with postmenstrual spotting with prior cesarean section. J Minim Invasive Gynecol. 2010;17(3):361-4.

9. Gubbini G, Casadio P, Marra E. Resectoscopic correction of the "isthmocele" in women with postmenstrual abnormal uterine bleeding and secondary infertility. J Minim Invasive Gynecol. 2008;15(2):172-5.

10. Thurmond AS, Harvey WJ, Smith SA. Cesarean section scar as a cause of abnormal vaginal bleeding: diagnosis by sonohysterography. J Ultrasound Med. 1999;18(1):13-6.

11. Gubbini G, Centini G, Nascetti D, Marra E, Moncini I, Bruni L, et al. Surgical hysteroscopic treatment of cesarean-induced isthmocele in restoring fertility: prospective study. J Minim Invasive Gynecol. 2011;18(2):234-7.

12. Perez-Medina T, Sancho-Sauco J, Rios M, Pereira A, Argila N, Cabezas E, et al. Hysteroscopy in pregnancy-related conditions: descriptive analysis in 273 patients. J Minimally Invasive Gynecol. 2014;21(3):41725.

13. Uppal T, Lanzarone V, Mongelli M. Sonographically detected caesarean section scar defects and menstrual irregularity. J Obstet Gynaecol. 2011;31(5):4136.

14. Melo-Cerda I. Cesarean scar defect. Ginecol Obstet Mex. 2014;82(8):530-4.

15. Erickson SS, Van Voorhis BJ. Intermenstrual bleeding secondary to cesarean scar diverticuli: report of three cases. Obstet Gynecol. 1999;93(5 Pt 2):802-5.

16. Tower AM, Frishman GN. Cesarean scar defects: an underrecognized cause of abnormal uterine bleeding and other gynecologic complications. J Minim Invasive Gynecol. 2013;20(5):562-72.

17. Monteagudo A, Carreno C, Timor-Tritsch IE. Saline infusion sonohysterography in nonpregnant women with previous cesarean delivery: the "niche" in the scar. J Ultrasound Med. 2001;20(10):1105-15.

18. Raimondo G, Grifone G, Raimondo D, Seracchioli R, Scambia G, Masciullo V. Hysteroscopic treatment of symptomatic cesarean-induced isthmocele: a prospective study. J Minim Invasive Gynecol. 2015;22(2):297-301.

19. Chang Y, Tsai EM, Long CY, Lee CL, Kay N. Resectoscopic treatment combined with sonohysterographic evaluation of women with postmenstrual bleeding as a result of previous cesarean delivery scar defects. Am J Obstet Gynecol. 2009;200(4):370 e1-4.

20. Roma Dalfo A, Ubeda B, Ubeda A, Monzon M, Rotger R, Ramos $R$, et al. Diagnostic value of hysterosalpingography in the detection of intrauterine abnormalities: a comparison with hysteroscopy. AJR Am J Roentgenology. 2004;183(5):1405-9..

21. Ahmadi F, Torbati L, Akhbari F, Shahrzad G. Appearance of uterine scar due to previous cesarean section on hysterosalpingography: various shapes, locations and sizes. Iran J Radiol. 2013;10(2):103-10.

22. Fabres C, Arriagada P, Fernandez C, Mackenna A, Zegers F, Fernandez E. Surgical treatment and follow-up of women with intermenstrual bleeding due to cesarean section scar defect. J Minim Invasive Gynecol. 2005;12(1):25-8. 
23. Florio $\mathrm{P}$, Filippeschi M, Moncini I, Marra E, Franchini M, Gubbini G. Hysteroscopic treatment of the cesarean-induced isthmocele in restoring infertility. Curr Opin Obstet Gynecol. 2012;24(3):180-6.

24. Marotta ML, Donnez J, Squifflet J, Jadoul P, Darii N, Donnez O. Laparoscopic repair of post-cesarean section uterine scar defects diagnosed in nonpregnant women. J Minim Invasive Gynecol. 2013;20(3):38691.

25. Donnez O, Jadoul P, Squifflet J, Donnez J. Laparoscopic repair of wide and deep uterine scar dehiscence after cesarean section. Fertil Steril. 2008;89(4):97480.

26. Florio P, Gubbini G, Marra E, Dores D, Nascetti D, Bruni L, et al. A retrospective case-control study comparing hysteroscopic resection versus hormonal modulation in treating menstrual disorders due to isthmocele. Gynecol Endocrinol. 2011;27(6):434-8.

27. Consenso de la Federación Colombiana de Obstetricia y Ginecología. Federación Colombiana de Perinatología. Racionalización del uso de la cesárea en Colombia. Consenso de la Federación Colombiana de Obstetricia y Ginecología (Fecolsog) y la Federación Colombiana de Perinatología (Fecopen). Bogotá, 2014. Rev Colombiana Obstet Ginecol. 2014;65(2):139-51.

28. Bij de Vaate AJ, Brölmann HA, van der Voet LF, van der Slikke JW, Veersema S, Huirne JA. Ultrasound evaluation of the cesarean scar: relation between a niche and postmenstrual spotting. Ultrasound Obstet Gynecol. 2011;37(1):93-9.

29. Zimmer EZ, Bardin R, Tamir A, Bronshtein M. Sonographic imaging of cervical scars after Cesarean section. Ultrasound Obstet Gynecol. 2004;23(6):594-8.

30. Ofili-Yebovi D, Ben-Nagi J, Sawyer E, Yazbek J, Lee C, Gonzalez J, et al. Deficient lower-segment fesarean section scars: prevalence and risk factors. Ultrasound Obstet Gynecol. 2008;31(1):72-7. 